\title{
Article \\ Functional Characterization of the Stipa purpurea P5CS Gene under Drought Stress Conditions
}

\author{
Danni Yang ${ }^{1,2,3,4,+}$, Ruize $\mathrm{Ni}^{1,2,3,4,+}$, Shihai Yang ${ }^{1,2}$, Yanan $\mathrm{Pu}^{1,2,3,4}$, Min Qian ${ }^{1,2,3,5}$, Yunqiang Yang $1,2,3, *$ \\ and Yongping Yang $1,2,3, *$
}

1 Plant Germplasm and Genomics Center, Kunming Institute of Botany, Chinese Academy of Sciences, Kunming 650201, China; yangdanni@mail.kib.ac.cn (D.Y.); niruize19@mails.ucas.ac.cn (R.N.); yang-shihai@163.com (S.Y.); puyanan94@163.com (Y.P.); qianmin181@163.com (M.Q.)

2 Institute of Tibetan Plateau Research at Kunming, Kunming Institute of Botany, Chinese Academy of Sciences, Kunming 650201, China

3 Key Laboratory of Tibetan Environment Changes and Land Surface Processes, Institute of Tibetan Plateau Research, Chinese Academy of Sciences, Beijing 100085, China

4 University of Chinese Academy of Sciences, Beijing 100049, China

5 Yunnan Population and Family Planning Science and Technology Research Institute, Kunming 650021, China

* Correspondence: yangyunqiang@mail.kib.ac.cn (Y.Y.); yangyp@mail.kib.ac.cn (Y.Y.)

+ These authors contributed equally to this work.

check for updates

Citation: Yang, D.; Ni, R.; Yang, S.; Pu, Y.; Qian, M.; Yang, Y.; Yang, Y. Functional Characterization of the Stipa purpurea P5CS Gene under Drought Stress Conditions. Int. J. Mol Sci. 2021, 22, 9599. https://doi.org/ 10.3390/ijms22179599

Academic Editors: Tomasz Hura,

Katarzyna Hura and

Agnieszka Ostrowska

Received: 16 July 2021

Accepted: 27 August 2021

Published: 4 September 2021

Publisher's Note: MDPI stays neutral with regard to jurisdictional claims in published maps and institutional affiliations.

Copyright: (c) 2021 by the authors. Licensee MDPI, Basel, Switzerland. This article is an open access article distributed under the terms and conditions of the Creative Commons Attribution (CC BY) license (https:/ / creativecommons.org/licenses/by/ $4.0 /)$.

\begin{abstract}
Free proline has multiple functions in plant cells, such as regulating osmotic potential and protecting both proteins and cell membranes. The expression of $\Delta 1$-Pyrroline-5-carboxylate synthase (P5CS), a key enzyme in the proline biosynthetic pathway, increases under drought, salt and cold stress conditions, causing plant cells to accumulate large amounts of proline. In this study, we cloned and identified the P5CS gene from Stipa purpurea, which has a full-length of $2196 \mathrm{bp}$ and encodes 731 amino acids. A subcellular localization analysis indicated that SpP5CS localized to the cytoplasm. The ectopic overexpression of SpP5CS in Arabidopsis thaliana resulted in higher proline contents, longer roots, higher survival rates and less membrane damage under drought stress conditions compared with wild-type controls. SpP5CS-overexpressing A. thaliana was more resistant to drought stress than the wild type, whereas the deletion mutant $s p 5 c s$ was less resistant to drought stress. Thus, SpP5CS may be a potential candidate target gene for increasing plant resistance to drought stress.
\end{abstract}

Keywords: Stipa purpurea; P5CS; proline; drought tolerance

\section{Introduction}

Almost half of the agricultural area in the world is at risk of drought, which has become the most important problem in global agriculture, seriously affecting crop yields [1]. Increasing crop yields under drought conditions is a major goal of plant breeding, and physiological and molecular mechanisms of plant drought resistance have been studied to develop targets for molecular breeding. Drought can cause plant cells to produce excess reactive oxygen species (ROS) and free radicals. The accumulation of ROS and free radicals leads to membrane lipid peroxidation and subcellular structure damage, which destroys the integrity of plant cell membranes [2]. Water is an important solvent and reaction substrate in cells, and many biological macromolecules become permanently deformed and functionless after dehydration. To survive, plants under drought stress conditions accumulate some low molecular weight metabolites called osmolytes, including proline, glycine betaine, mannitol and quaternary amines. These osmolytes act as solutes to reduce the water potential in cells to improve the water absorption or water retention capacity of the cell while maintaining the cell volume and protecting the cell membrane [3].

The amino acid proline, as a common osmolyte, has many effects on plant cells under drought conditions. Under osmotic stress conditions, large quantities of proline accumulate in plant cells and the proline accumulation level varies among species, with levels in some 
species increasing 100-fold compared with the levels under normal conditions [4]. For example, sunflower (Helianthus annus) seeds accumulate more proline under drought or salt damage [5]. The accumulation of proline also responds to the induction of abscisic acid (ABA) and polyamines. The proline content of wheat increased significantly after treatment with exogenous ABA or polyamines [6,7]. The increase in free proline reduces the intracellular osmotic potential and maintains the intracellular water content. Under osmotic stress, proline can increase the activity of antioxidant enzymes [8]. Proline also acts as a molecular chaperone to retain and stabilize protein three-dimensional structures, which are required to maintain their normal functions [4]. A high proline level in a cell reduces the damage caused by free radicals, scavenges ROS and balances the cytoplasmic $\mathrm{pH}$ and redox states, thereby maintaining the basic metabolism of the cell $[9,10]$. Proline also stabilizes the internal membrane and helps maintain the integrity of cell structures and functions. Finally, proline aids in storing carbon and nitrogen [11].

$\Delta 1$-Pyrroline-5-carboxylate synthase (P5CS, E.C. 2.7.2.11, E.C. 1.2.1.41) is a key enzyme in the proline biosynthetic pathway, which regulates the proline contents in plant cells and improves resistance to osmotic stress. The major precursor of proline synthesis under adverse conditions is glutamic acid, which is catalyzed by P5CS and $\Delta 1$-Pyrroline5-carboxylate reductase into proline [12]. Under stress conditions, proline accumulations in plant cells are mainly regulated by the enzyme P5CS. Studies in Hordeum vulgare, Oryza sativa and other plants have shown that the P5CS expression and activity levels are upregulated under drought and salt stress conditions, thus increasing the proline content [13-16]. As a rate-limiting enzyme in the proline biosynthetic pathway, P5CS regulates proline biosynthesis through feedback inhibition and transcription regulation [17]. Most plants have two P5CS isoforms, such as Brassica napus, Phaseolus vulgaris and O. sativa [18-20]. Recent studies have found three P5CS genes in Lilium regale and Medicago truncatula [21,22]. The transcriptional regulation mechanism of different isoforms of P5CS genes varies from species to species. In O. sativa, OsP5CS1 is a housekeeping gene, and OsP5CS2 responds to stress [20]. In B. napus, BnP5CS1 and BnP5CS2 are both induced by salt, ABA [18]. In M. truncatula, the expression levels of MtP5CS2 and MtP5CS3 increase under salt and drought stress, while the expression of MtP5CS1 is constitutive [22]. From the overexpression of PvP5CS1 and PvP5CS2 from P. vulgaris in Arabidopsis thaliana, both genes increased the proline content and salt tolerance, but PvP5CS2 had a stronger ability to synthesize proline under salt stress [19]. The overexpression of P5CS increased the tolerance to drought and salt stress in transgenic rice, tobacco and wheat by increasing the proline content [23-25]. The overexpression of A. thaliana P5CS in tobacco increases the transgenic tobacco's proline content and enhances its resistance to osmotic stress compared with the controls. The P5CS gene from Vigna aconitifolia, under the control of the abscisic acid-inducible promoter complex, enhances the salt stress tolerance of transgenic sugarcane [26].

Stipa purpurea is a constructive species of alpine grass found on the Qinghai-Tibet Plateau. It readily survives in extremely arid areas and has a strong drought tolerance. Additionally, S. purpurea plays an important role in protecting the soil and is used as forage [27]. In this study, we cloned the P5CS gene from S. purpurea and measured the expression levels of this gene under soil drought, cold and salt conditions. Additionally, SpP5CS overexpression in A. thaliana verified its function in drought stress. An analysis of several phenotypes and physiological indicators showed that the ectopic expression of SpP5CS in A. thaliana leads to greater stress resistance compared with the WT. Thus, $S p P 5 C S$ may be an important gene in the drought resistance mechanism of $S$. purpurea.

\section{Results}

\subsection{Cloning and Structural Analysis of SpP5CS}

Based on the EST sequence obtained by transcriptome sequencing, we used RACE (rapid amplification of cDNA ends) technology to obtain the CDS sequence of SpP5CS. The SpP5CS (GenBank: MZ710734) contains an open reading frame of $2196 \mathrm{bp}$ that encodes 
731 amino acids. The molecular weight of the SpP5CS protein is $78.95 \mathrm{kDa}$, and the theoretical isoelectric point is 5.95. The SMART protein structural analysis of SpP5CS revealed two functional domains: $\gamma$-glutamyl kinase (GK) and glutamic- $\gamma$-semialdehyde dehydrogenase (GSA DH) (Figure 1a). To analyze the relationship between SpP5CS and P5CS genes from other species, we extracted the P5CS gene sequences of Brachypodium distachyon, Lolium perenne, Triticum aestivum, Aegilops tauschii, O. sativa, Oryza brachyantha, Zea mays, Sorghum bicolor, A. thaliana, Helianthus tuberosus, L. regale, M. truncatula, P. vulgaris, B. napus and Setaria italica through the BLAST tool of NCBI (https:/ /blast.ncbi.nlm.nih. gov / Blast.cgi, accessed on 6 August 2021). A phylogenetic tree was constructed using the BLAST tool of MEGA X and the neighbor-joining method. SpP5CS is highly similar to P5CS from B. distachyon (Figure 1b). The results indicate that SpP5CS belongs to the P5CS2 isoform.

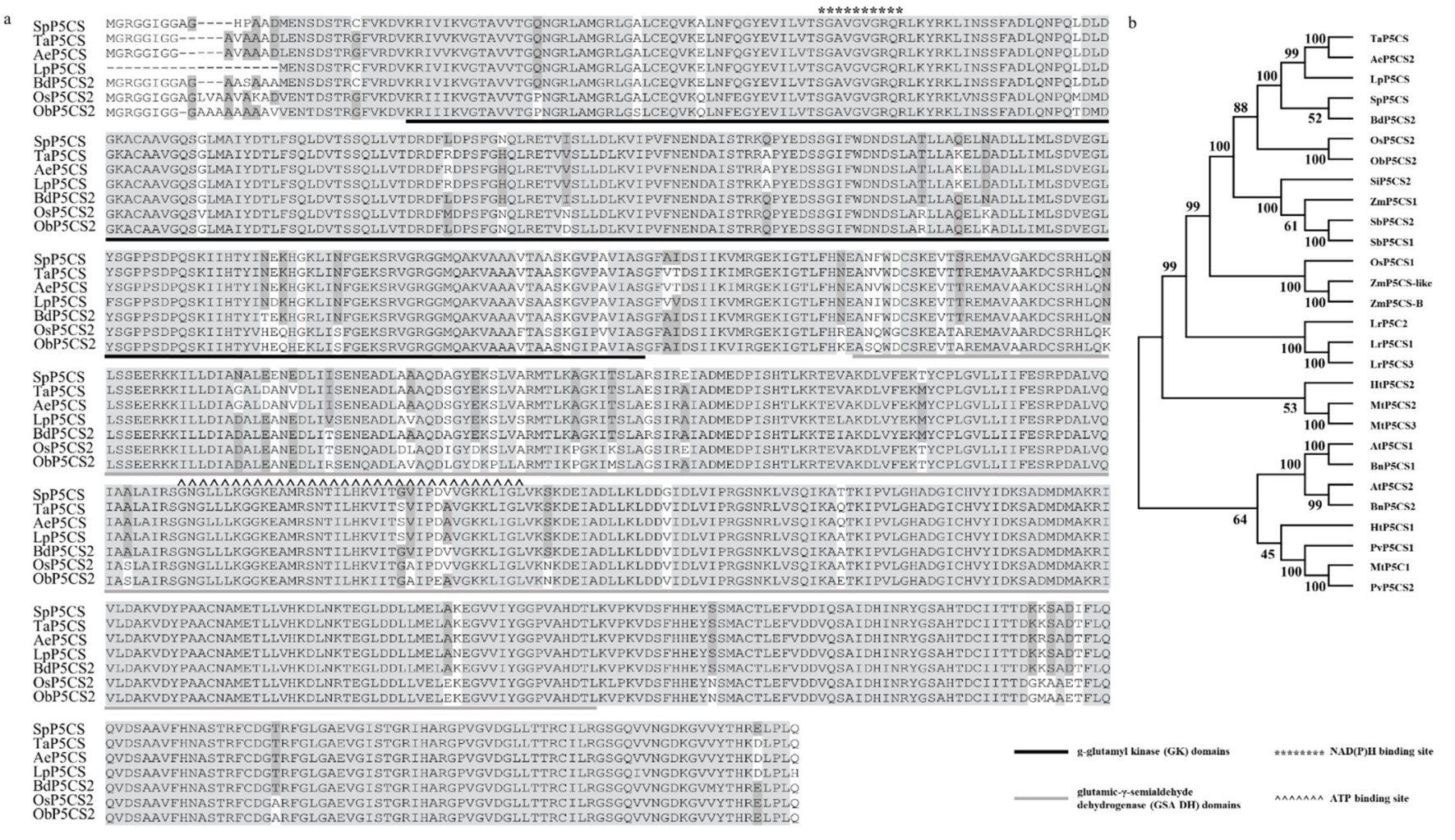

Figure 1. Sequences analysis of SpP5CS. (a) Multiple sequence alignment of SpP5CS and homologous proteins from other species. Legend shows the $\gamma$-glutamyl kinase (GK; 30-275 bp) and glutamic- $\gamma$-semialdehyde dehydrogenase (GSA DH; 299-600 bp) domains; (b) A phylogenetic tree constructed using SpP5CS and P5CS proteins from other plant species. BdP5CS2 (B. distachyon, XP_003564608.1), LpP5CS (L. perenne, AGQ04179.1), TaP5CS (T. aestivum:KAF7024610.1), AeP5CS2 (A. tauschii, XP_020198194.1), OsP5CS1 (O. sativa, O04226.2), OsP5CS2 (O. sativa, BAB64280.1), ObP5CS2 (O. brachyantha, XP_006645010.2), ZmP5CS1 (Z. mays, AQK99427.1), ZmP5CSB(Z. mays, AQK86404.1), ZmP5CSB-like (Z. mays, NP_001339256), SbP5CS1 (S. bicolor, ACU65226.1), SbP5CS2 (S. bicolor, ACU65227.1), AtP5CS1 (A. thaliana, P54887.1), AtP5CS2 (A. thaliana, P54888.1), HtP5CS1 (H. tuberosus, AHJ08569.1), HtP5CS2 (H. tuberosus,AHJ08570.1), LrP5CS1 (L. regale, KT972374), LrP5CS2 (L. regale, KT972375), LrP5CS3 (L. regale, KT972376), MtP5CS1 (M. truncatula, CAC82184.1), MtP5CS2 (M. truncatula, AET87351.1), MtP5CS3 (M. truncatula, AET35478.1), PvP5CS1 (P. vulgaris, ABY61079.1), PvP5CS2 (P. vulgaris, ABY89287.1), BnP5CS1 (B. napus, AF314811), BnP5CS2 (B. napus, AF314812) and SiP5CS2 (S. italica, XP_004970573.1).

\subsection{The Expression of SPP5CS and the Proline Content Analysis}

qRT-PCR was used to detect the expression of the SpP5CS in S. purpurea leaves after exposure to different treatments. After growing for 2 weeks, S. purpurea was exposed to different abiotic stress conditions (cold, salt, PEG and soil drought) for different times. After $6 \mathrm{~h}$ of the cold-stress treatment at $4{ }^{\circ} \mathrm{C}, \operatorname{SpP} 5 \mathrm{CS}$ expression increased to two times that of the control, but after $12 \mathrm{~h}$ of continuous treatment, the expression level decreased to 1.7 times that of the control. Additionally, after being treated with $150-\mathrm{mM} \mathrm{NaCl}$ for $6 \mathrm{~h}$, 

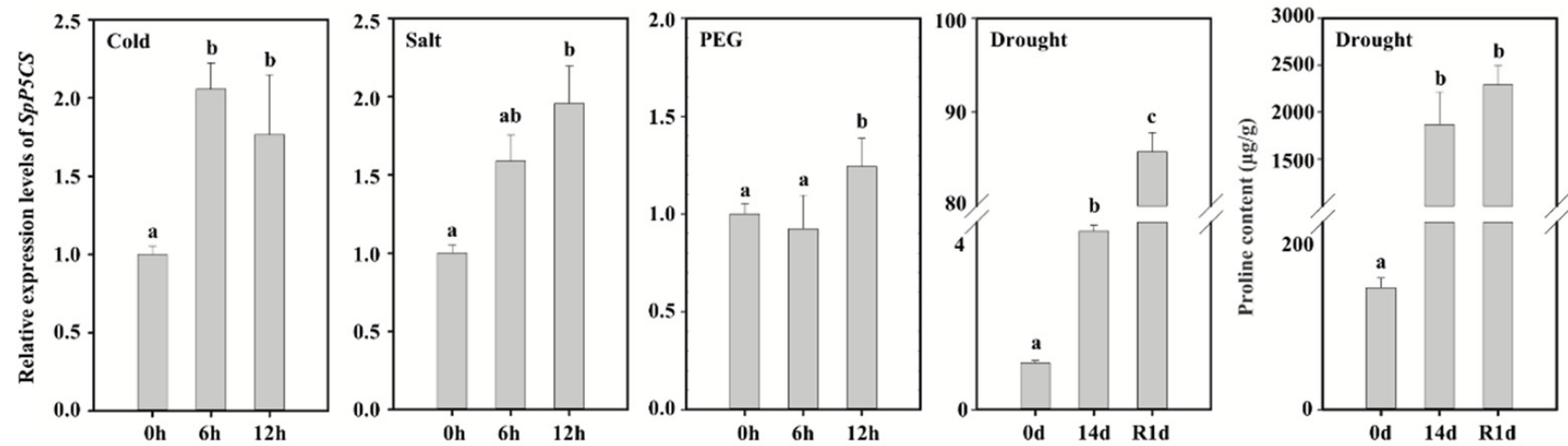

Figure 2. qPCR analysis of the expression levels of SpP5CS under cold, salt, drought and soil drought stress conditions. The experiment has three biological replicates, and the error bar denotes the standard deviation. The data are analyzed by one-way ANOVA (Tukey's test). Different letters indicate significant differences among the relative expression levels of the genes at $p<0.05$.

\subsection{Identification of Ectopically Expressed 35S:SpP5CS-GFP Transgenic Plants}

To further explore SpP5CS's functions, we cloned the gene and transformed it into A. thaliana, with the expression being driven by the $35 \mathrm{~S}$ Cauliflower mosaic virus promoter. The primers SpP5CS-GFP-F and SpP5CS-GFP-R were used to successfully identify the transgenic plants (Figure 3a). We received six transgenic lines, and the transformation efficiency was about $0.1 \%$. We observed the SpP5CS-GFP fusion protein in the cytoplasm from transgenic $A$. thaliana using a laser confocal microscope (Figure $3 b$ ).

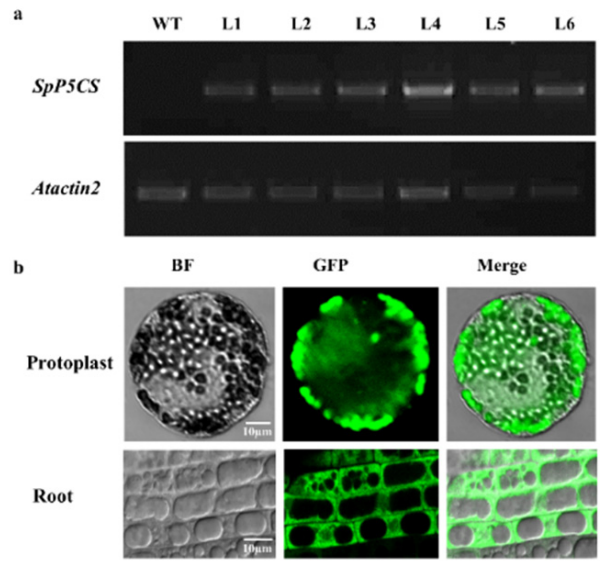

Figure 3. RT-PCR identification of SpP5CS and the subcellular localization of the 35S:SpP5CS-GFP fusion protein. (a) The RT-PCR identification of lines 1-6 of OE-SpP5CS A. thaliana; (b) Subcellular localization of the 35S:SpP5CS-GFP fusion protein in the root tips and leaf mesophyll protoplasts of transgenic A. thaliana. 


\subsection{SpP5CS Overexpression Enhances Drought Tolerance}

To analyze whether SpP5CS expression enhances the plant's drought resistance function, the SpP5CS gene was first transformed into a P5CS deletion mutant to obtain a $p 5 c s / S p P 5 C S$ complemented plant. Then, the WT, mutant, $p 5 c s / S p P 5 C S-c o m p l e m e n t e d$ and OE-SpP5CS A. thaliana seeds were cultured on 1/2 Murashige and Skoog (MS) medium supplemented with 5\% PEG 6000 and ordinary 1/2 MS medium, and the phenotypes were observed. On 1/2 MS medium containing 5\% PEG 6000, the average root length of the OE-SpP5CS plants was $2.17 \mathrm{~cm}$, and the average root length of a mutant plants was $0.69 \mathrm{~cm}$. The root lengths of OE-SpP5CS plants under drought conditions were significantly longer than those of the other types of plants, whereas the mutant plants themselves were significantly shorter than the other types of plants (Figure $4 a, b)$. The average root lengths were $1.18 \mathrm{~cm}$ and $1.54 \mathrm{~cm}$, respectively, on $1 / 2 \mathrm{MS}$ medium, with no significant differences among the root lengths of the various plants.
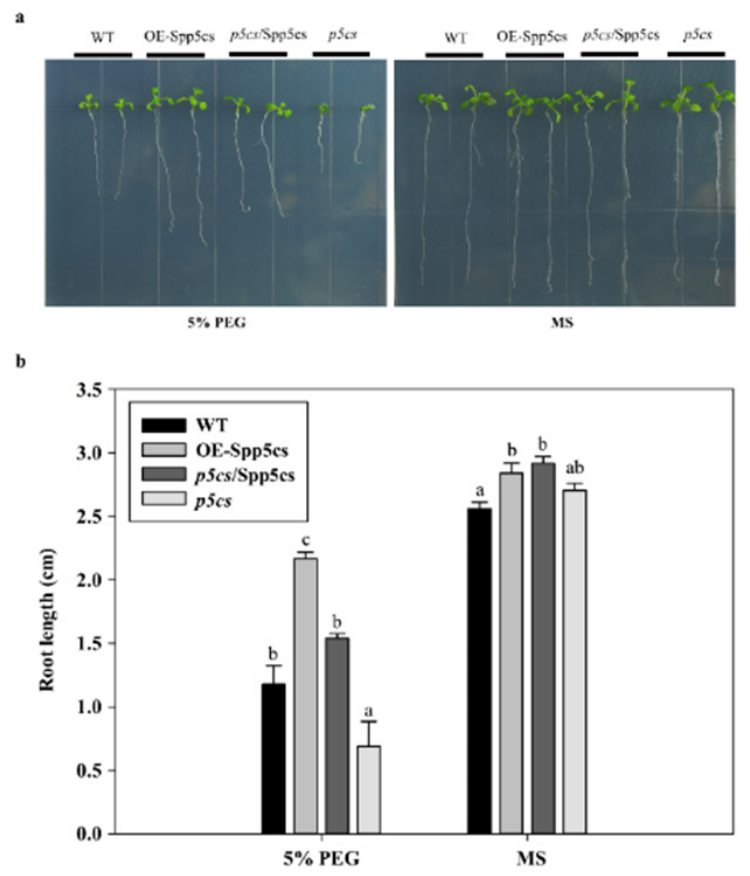

Figure 4. Root length analysis of WT, OE-SpP5CS, $p 5 c s /$ SpP5CS-complemented and $p 5 c s$ mutant $A$. thaliana seedlings in response to drought stress. (a) Growth of plants under drought stress conditions. Seeds were grown on the 1/2 MS medium and 1/2 MS medium supplemented with 5\% PEG 6000 for $10 \mathrm{~d}$; (b) Root lengths of WT, OE-SpP5CS, p5cs/SpP5CS-complemented and $p 5 c s$ mutant plants grown under normal and drought stress conditions. Data were obtained from three independent experiments. Each column represents the mean $\pm \mathrm{SD}$, and the error bar denotes the standard deviation. Different letters indicate significant differences in the root length (one way ANOVA, Tukey's test, $p<0.05)$.

To further identify the functions of SpP5CS in improving plant drought resistance, WT, mutant, $p 5 c s / S p P 5 C S-c o m p l e m e n t e d$ and OE-SpP5CS A. thaliana seedlings were planted in square plastic pots and subjected to the soil drought treatment. After $15 \mathrm{~d}$ of the drought treatment, the growth status of OE-SpP5CS A. thaliana was the best among all the plant types (Figure $5 \mathrm{a}$ ). Then, their survival rates and electrical conductivity levels were measured. As shown in Figure $5 c$, after $5 \mathrm{~d}$ of drought treatment, the survival rate of OE-SpP5CS A. thaliana was $87.4 \%$, and the plants showed the lowest degree of damage at $6.4 \%$, while the survival rate of the mutant $A$. thaliana was $66.1 \%$, and the plants showed the greatest degree of damage at $33 \%$. After $12 \mathrm{~d}$ of drought treatment, the survival rate of OE-SpP5CS A. thaliana was $63.6 \%$, and the damage degree was $36.4 \%$, while the survival rate of the mutant $A$. thaliana was $25.8 \%$, and the damage degree was $74.2 \%$. After $15 \mathrm{~d}$ of drought treatment, the survival rate of the OE-SpP5CS A. thaliana was $56.1 \%$, and the 
damage degree was $43.9 \%$, while the survival rate of the mutant $A$. thaliana was $13.7 \%$, and the damage degree was $86.3 \%$ (Figure $5 b, c$ ). The survival rates and degrees of damage for the WT and $p 5 c s / S p P 5 C S$-complemented $A$. thaliana were at intermediate levels, with little differences between the two lines. Therefore, the overexpression of SpP5CS improved the drought resistance of transgenic $A$. thaliana.

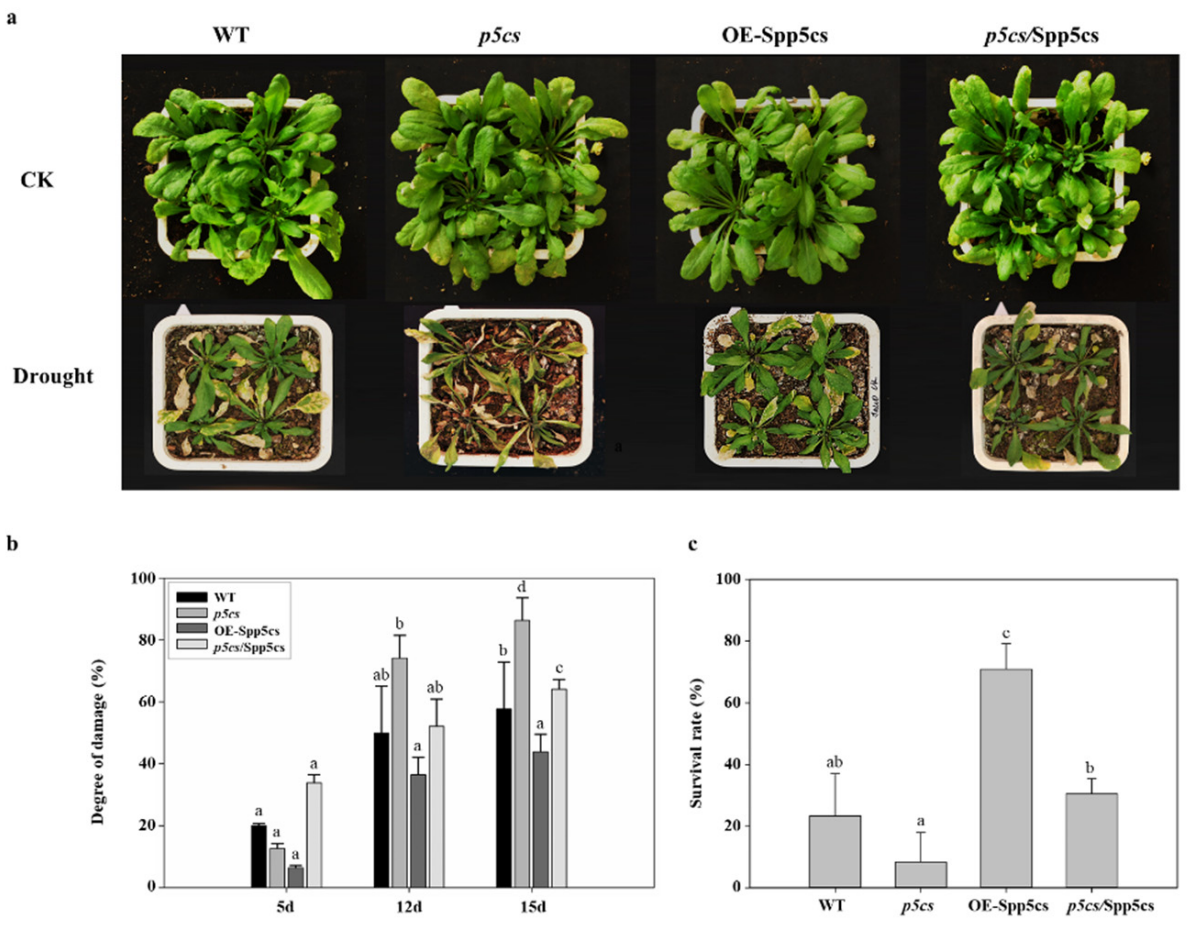

Figure 5. Phenotypic and membrane damage analyses of WT, OE-SpP5CS, p5cs/SpP5CScomplemented and $p 5 c s$ mutant $A$. thaliana plants. (a) Phenotypes of WT, OE-SpP5CS, p5cs/SpP5CScomplemented and $p 5 c s$ mutant plants in response to 12 days of soil drought stress. $\mathrm{CK}$ is the control group under the condition of normal irrigation; (b) Comparison of the degree of membrane damage among WT, SpP5CS, p5cs/SpP5CS-complemented and $p 5 c s$ mutant plants in response to soil drought stress; (c) Comparison of the survival rates among WT, SpP5CS, p5cs/SpP5CS-complemented and p5cs mutant plants after 12 days of soil drought stress. The soil drought treatment adopts continuous withholding water. The experiment has three biological and technical replicates. The data are expressed as the mean \pm SD from three independent experiments. Different letters indicate significant differences in the degree of damage or survival rate (one-way ANOVA, Tukey's test, $p<0.05$ ).

\subsection{Variation in the Proline Content}

To investigate the effects of SpP5CS, the proline content was determined for WT, $p 5 c s$ mutant, $p 5 c s$ /SpP5CS-complemented and OE-SpP5CS A. thatiana using the acidicninhydrin assay. After $5 \mathrm{~d}$ of drought treatment, the proline content of the OE-SpP5CS A. thaliana was significantly higher than the other plant types and more than five times that of the WT. At this time, the proline content of the mutant was the lowest among all the plants at approximately half the WT value. After $12 \mathrm{~d}$ of drought treatment, the proline content of the OE-SpP5CS A. thaliana was still the highest, but the differences compared with the WT decreased. The mutant still had the lowest content. After $15 \mathrm{~d}$ of drought treatment, the proline contents of the plants were nearly the same (Figure 6). These results indicate that the SpP5CS gene improves the drought resistance of plants by increasing their proline content. 


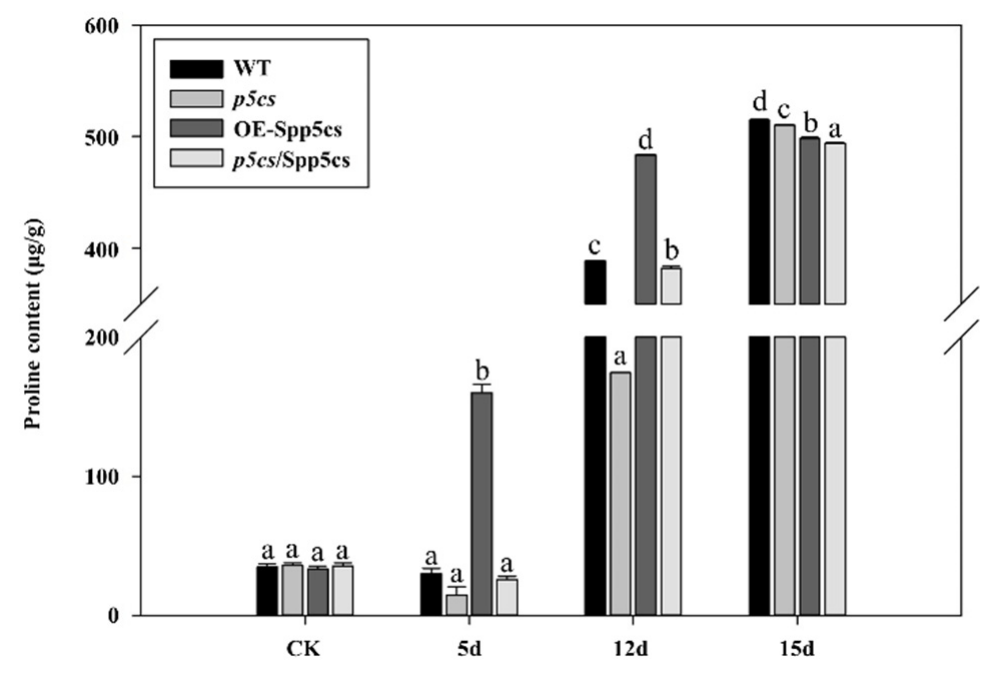

Figure 6. Proline contents of WT, OE-SpP5CS, $p 5 c s / S p P 5 C S$-complemented and $p 5 c s$ mutant plants after the soil drought treatment. The soil drought treatment adopts continuous withholding water. CK is the control group under the condition of normal irrigation. The experiment has three biological and technical replicates. Data are the mean values \pm SD obtained from three independent experiments (error bars $=\mathrm{SD}, n=15$ ). Different letters indicate significant differences in the proline content (one-way ANOVA, Tukey's test, $p<0.05$ ).

\section{Discussion}

\subsection{Molecular Characterization of SpP5CS}

As an important gene involved in proline synthesis, P5CS has been reported in O. sativa, P. vulgaris, A. thaliana and S. bicolor, and it contains the conserved domains GK and GSA DH $[28,29]$. In this study, we cloned the full-length CDS sequence of SpP5CS gene from S. purpurea. Then, we performed multiple sequence alignment and phylogenetic analysis of the P5CS protein of S. purpurea and other species. We found that they have similar structural domains (GSA DH and GK), and SpP5CS and P5CS2 of multiple species are in the same branch. Due to their structural similarity, SpP5CS may have functions similar to P5CS2 in other species. According to previous reports, the research of P5CS2 mainly focused on growth and development, drought resistance and salt resistance [18-22,30]. We speculate that SpP5CS has a similar effect. Previous subcellular localization studies have shown that P5CS2 are localized in the cytoplasm [30]. The subcellular localization results show that SpP5CS is localized in the cytoplasm, which is consistent with the previous report.

\subsection{Expression Analysis of SpP5CS}

The gene expression analysis shows differences in the gene expressions under different environmental conditions, which helps predict the gene function. The P5CS gene responds to osmotic stress and increases the proline content of plants to improve osmotic resistance. In S. italica, SiP5CS2 is remarkably upregulated after $9 \mathrm{~d}$ of drought treatment [31]. After $10 \mathrm{~d}$ of salt stress, the expression of Opuntia streptacantha P5CS increases [32]. Salt-tolerant varieties of $O$. sativa have more OsP5CS mRNA transcripts and proline under high-salt than normal conditions [14]. The expression of Setaria viridis P5CS2 is upregulated after 6 and $10 \mathrm{~d}$ of an osmotic stress treatment in the form of an external PEG 8000 application [33]. In addition to the response to osmotic stress, the P5CS gene is also upregulated during cold stress. After $24 \mathrm{~h}$ of a cold treatment at $4{ }^{\circ} \mathrm{C}$, the transcription level of the P5CS gene in L. perenne is significantly upregulated [34]. The expression of SpP5CS was significantly upregulated under soil drought, cold and salt stress, which was consistent with the results of the other species. According to reports, different isoforms of P5CS genes are induced by different stresses. In Sorghum bicolor, the transcription levels of SbP5CS1 and SbP5CS2 are both upregulated under drought and salt stress. Meanwhile, the transcription level of SbP5CS2 increased faster and more obviously than that of SbP5CS2 under the induction of 
methyl jasmonic acid [29]. In our study, SpP5CS strongly responds to soil drought, which may be the result of $S$. purpurea adapted to arid environment for a long time. In addition, the proline content of S. purpurea increased significantly after 14 days of soil drought and 1 day after rehydration. We speculate that $S$. purpurea accumulates proline by increasing the expression of the SpP5CS gene to improve the drought resistance after drought stress.

\subsection{Potential Mechanism of SpP5CS for Improving Plant Resistance to Drought Stresses}

There are various drought resistance mechanisms in plants, among which, an increasing root length and increasing osmotic pressure regulatory substances are very common. As a key enzyme in the proline biosynthesis pathway, P5CS also enhances root growth, and it plays an important role in plant resistance to drought. The electrical conductivity of cell leakage indirectly evaluates the degree of cell membrane damage [35]. OE-SpP5CS A. thaliana showed the lowest degree of membrane damage compared with WT. P5CS transgenic rice increase their proline contents under water shortage conditions and have increased fresh shoot weights, by 50-95\%, after PEG treatments [23]. The overexpression of SpP5CS in A. thaliana increases the proline content to combat drought stress. Combining the results of the heterologous expression in A. thaliana and qPCR in S. purpurea, SpP5CS can enhance the drought resistance of plants by increasing the proline production.

S. purpurea is widely distributed in the precipitation gradient of the Qinghai-Tibet Plateau, and it can still survive in extremely arid areas. Previous studies have revealed that S. purpurea in arid regions have a higher content of proline than S. purpurea in humid regions, and a transcriptome analysis has shown that the P5CS gene is upregulated at a higher level in arid regions [36]. The proteomic analysis also suggests that the P5CS protein in S. purpurea from more arid areas has a high level of upregulation [37]. Furthermore, we verified the single gene function of SpP5CS to show that SpP5CS improves the drought resistance of plants. These studies all indicate that the upregulation of SpP5CS gene leads to an increase in the proline content to enhance osmotic regulation, which is an important part of the drought-resistant mechanism of S. purpurea.

\section{Materials and Methods}

\subsection{Plant Materials}

Mature S. purpurea seeds were collected from Nyima County, Tibet $\left(32^{\circ} 00^{\prime} 05^{\prime \prime} \mathrm{N}\right.$, $\left.86^{\circ} 50^{\prime} 54^{\prime \prime} \mathrm{E}\right)$, China. The seeds were planted in square plastic pots $(10 \mathrm{~cm} \times 10 \mathrm{~cm} \times 8 \mathrm{~cm})$ containing nutrient soil (Pindstrup Mosebrug A/S, Ryomgaard, DK) and placed in the dark to germinate. After that, the seedlings were moved into a greenhouse and grown for about 2 weeks (trefoil stage) for cold and soil drought treatments. After 0,6 , and $12 \mathrm{~h}$ of cold treatment in a plant incubator (SAFU EXPERIMENTAL APPARATUB TECHNOLOGY, China) at $4{ }^{\circ} \mathrm{C}$, the second and third leaves of $S$. purpurea were collected. For the soil drought treatment, the second and third leaves of $S$. purpurea were selected after continuously withholding water for $0 \mathrm{~d}$ and $14 \mathrm{~d}$ and rehydration (fully watered) for 1 day. For salt and PEG treatment, S. purpurea seeds were soaked in water overnight and germinated on moist filter paper in the dark. After germination, the seedlings were transplanted into a plastic pot $(10 \mathrm{~cm} \times 10 \mathrm{~cm} \times 8 \mathrm{~cm})$ containing the same amount of vermiculite and $1 / 2$ liquid MS in a greenhouse for about 2 weeks (trefoil stage). After that, the $1 / 2$ MS was replaced with $1 / 2 \mathrm{MS}$ containing $150-\mathrm{mM} \mathrm{NaCl}$ or $30 \%$ polyethyleneglycol 6000 (PEG 6000). After 0,6 and $12 \mathrm{~h}$ of salt or PEG treatment, the second and third leaves of S. purpurea were collected [38,39]. The samples were frozen in liquid nitrogen and stored at $-80{ }^{\circ} \mathrm{C}$ for subsequent RNA isolation. A thaliana plants (ecotype: Columbia ecotype, Col-0) were cultured for ectopic expression experiments on $1 / 2$ solid MS medium. After 10 days, the seedlings were transferred to nutrient soil. The $p 5 c s$ mutant (SALK_045245) was purchased from The Arabidopsis Information Resource (https: / /www.arabidopsis.org/, accessed on 11 June 2016). All the plants were grown in a greenhouse at $21^{\circ} \mathrm{C}$, with $75-80 \%$ relative humidity and a light intensity of 200- $\mu \mathrm{mol}$ photons $\mathrm{m}^{-2} \mathrm{~s}^{-1}$, under a 16-h light/8-h dark 
photoperiod. For untreated A. thaliana and S. purpurea, the normal irrigation situation was $30 \mathrm{~mL}$ of water every two days [37].

\subsection{RNA Isolation and SpP5CS Gene Cloning}

Total RNA was isolated from leaves of S. purpurea using an Eastep ${ }^{\circledR}$ Super Total RNA Extraction Kit (Promega, Madison, WI, USA), and the DNA digested with DNase I. RNA was quantified using a NanoDrop1000 (NanoDrop Technologies, Wilmington, DE, USA), and the integrity was checked on a $0.8 \%$ agarose gel. Then, RNA (5 $\mu \mathrm{g})$ was reverse-transcribed into first-strand cDNA using the GoScript ${ }^{\mathrm{TM}}$ Reverse Transcription System (Promega, Madison, WI, USA). We used RACE technology to obtain the full length of the SpP5CS sequence. Based on the transcriptome data (the Genome Sequence Archive in the BIG Data Center, Beijing Institute of Genomics (BIG), accession numbers CRA002018), the required EST fragments were screened out, and specific primers SpP5CS-RACE-1 (5'-TCTCGCGAAGCTGGTTCCCAAAACT-3') and SpP5CSRACE-2 (5'-GGGCCTGTTGCACATGACACACTGAA-3') were designed. Then we used SMART RACE cDNA kit (BD Biosciences Clontech, MountainView, CA, USA) to amplify the $5^{\prime}$ and $3^{\prime}$ sequences of the target gene. Then specific primer SpP5CS-F ( $5^{\prime}-$ ATGGGTAGAGGAGGGATCGGA- $\left.3^{\prime}\right)$ and SpP5CS-R (5'-TCATTGCAACGGAAGCTCCCT GTGG-3') were used to obtain the full-length CDS sequence of SpP5CS. We constructed the full length of SpP5CS into the pMD 18-T vector (Takara Bio, Kusatsu, Japan), and applied Sanger sequencing for paired-end sequencing.

\subsection{Sequence Analysis of the SpP5CS Gene}

The full-length SpP5CS cDNA sequence was translated into a protein using DNAMAN (Lynnon Biosoft, San Ramon, CA, USA). The protein was analyzed using the online SMART tool (http:/ / smart.embl-heidelberg.de/smart/show_motifs.pl, accessed on 5 October 2018) and ExPASy ProtParam tool (https:/ / web.expasy.org/protparam/, accessed on 8 October 2018). The protein sequences of other species were obtained using the BLAST tool of NCBI and aligned using the Clustal $X$ program [40]. Then, a maximum parsimony analysis was performed using MEGA $X$ with the neighbor-joining method and the 1000 bootstrap test replicates [41].

\subsection{Gene Expression Analysis}

To quantify the SpP5CS expression levels under different abiotic stress conditions, the cDNAs of processed S. purpurea samples were used as templates for quantitative real-time (qRT)-PCR analyses. The ABI 7500 Fast Real-time PCR System (Thermo Fisher Scientific, Waltham, MA, USA) and FastStart Universal SYBR Green Master (Roche, Indianapolis, IN, USA) were used. The reaction parameters for thermal cycling were as follows: $95^{\circ} \mathrm{C}$ for $10 \mathrm{~min}$, followed by 40 cycles of $94^{\circ} \mathrm{C}$ for $5 \mathrm{~s}$ and $60^{\circ} \mathrm{C}$ for $15 \mathrm{~s}$. The reaction system (total $20 \mu \mathrm{L}$ ) was as follows: cDNA template (dilute 5 times) $2 \mu \mathrm{L}$, nuclear-free water $6.8 \mu \mathrm{L}$, primer $(10 \mu \mathrm{M}) 0.6 \mu \mathrm{L}$, SYBR Green Master $10 \mu \mathrm{L}$. qRT-PCR was conducted in triplicate with different $c \mathrm{DNAs}$ synthesized from three biological replicates. The genespecific primer SpP5CS-qPCR-F/-R (SpP5CS-qPCR-F: 5'-GGTGATTCTGGTCACCTCCG-3', SpP5CS-qPCR-R: $5^{\prime}$-CCTTCCCATCCAGGTCCAAC-3') was used to amplify SpP5CS, with SpACT2 (F: 5'-CTGTGCCAATCTACGAGGGT-3' and R: 5'-GAACCACCGATCCAGACACT$\left.3^{\prime}\right)$ as the internal control. The data were analyzed using the $2^{-\triangle \triangle \mathrm{Ct}}$ method [42].

\subsection{Generation and Screening of Transgenic A. thaliana Plants}

The full-length SpP5CS CDS was inserted into the vector pRI 101 green fluorescent protein (GFP) and driven by the 35S Cauliflower mosaic virus promoter. Then, the recombinant plasmid was transformed into Agrobacterium tumefaciens stain GV3101 that was used to transform WT and $p 5 c s$ mutant $A$. thaliana by the floral dip method [43]. The harvested seeds were grown on MS medium supplemented with $50-\mathrm{mg} \mathrm{L}^{-1}$ kanamycin to select for positive transgenic plants. The transgenic $A$. thaliana were examined by RT-PCR using 
SpP5CS-GFP-F (5'-GACCCCGGGGGTACCGGATCCATGGGTAGAGGAGGGATCGGA-3') and SpP5CS-GFP-R (5'-TCAGAATTCGGATCCTCATTGCAACGGAAGCTCCCTGTGG-3'). The $A$. thaliana leaves of the same period were taken to extract RNA and reverse to obtain cDNA (according to the method in Section 4.2). Atactin2 (NM_001338358) were used to normalize the $A$. thaliana samples. The PCR reaction was performed under following conditions: 25 cycles at $95^{\circ} \mathrm{C}$ for $30 \mathrm{~s}, 55^{\circ} \mathrm{C}$ for $30 \mathrm{~s}, 72{ }^{\circ} \mathrm{C}$ for $2 \mathrm{~min}$.

\subsection{Subcellular Localization of SpP5CS}

The SpP5CS-overexpression (OE-SpP5CS) and WT A. thaliana lines were germinated on $1 / 2 \mathrm{MS}$ medium until the root lengths were approximately $2 \mathrm{~cm}$, and then, the roots were cut for subcellular localization. Leaf mesophyll protoplasts were isolated from ten-day-old cotyledons of OE-SpP5CS transgenic $A$. thaliana. The method of protoplast isolation was based on previous research [44]. A laser scanning confocal microscope (Olympus Optical, Tokyo, Japan) was used to observe the GFP expression.

\subsection{Drought Stress, Survival Rate, and Root-Length Measurements}

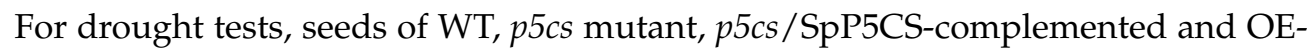
SpP5CS A. thaliana plants were sown on $1 / 2$ MS medium and $1 / 2$ MS medium supplemented with 5\% PEG 6000. Root lengths were measured after $10 \mathrm{~d}$. To determine the transgenic phenotypes under drought and normal irrigation conditions $(30 \mathrm{~mL}$ of water every two days), WT, mutant, $p 5 c s / S p P 5 C S$-complemented and OE-SpP5CS A. thaliana seedlings were planted in plastic pots filled with nutrient soil and grown in a greenhouse under normal irrigation conditions. Four-week-old seedlings with about 17 true leaves were used for drought stress treatment. Drought conditions were simulated by withholding water for 5,12 or $15 \mathrm{~d}$. After each treatment, the plants were sampled, quickly frozen in liquid nitrogen and stored in a refrigerator at $-80{ }^{\circ} \mathrm{C}$ for subsequent experiments. During the drought treatment process, the survival rate of $A$. thaliana at each drought stage was calculated.

\subsection{Relative Electrical Conductivity Measurement}

The relative conductivity of $A$. thaliana at different treatment periods was measured simultaneously. The fresh leaves (4th to 6 th leaves) were weighed (about $0.3 \mathrm{~g}$ ), cut into $0.5-\mathrm{mm}$ lengths and placed in a tube containing $10 \mathrm{~mL}$ of distilled water. To extract the air between the cells, the leaves in the distilled water were pumped in a vacuum for $20 \mathrm{~min}$. Then, the samples were placed at room temperature for $1 \mathrm{~h}$, and the conductivity of the solution were measured (S1). The solution was then heated to $100^{\circ} \mathrm{C}$ for $10 \mathrm{~min}$, cooled to room temperature and the final electrical conductivity (S2) was measured. The relative electrical conductivity (REC) was calculated as follows: REC $=\mathrm{S} 1 / \mathrm{S} 2$. The degree of damage was calculated as follows: (REC TREATMENT $\left.-\mathrm{REC}_{\mathrm{CONTROL}}\right) /\left(1-\mathrm{REC}_{\mathrm{CONTROL}}\right)$ $\times 100 \%$. The measurement method was based on the previously described method $[35,45]$.

\subsection{Proline Content Measurement}

The proline content was measured by a previously described method [46]. The sample was weighed (about $0.4 \mathrm{~g}$ ) and put in a 10-mL centrifuge tube containing $5 \mathrm{~mL}$ of $3 \%(w / v)$ 5-sulfosalicylic acid dihydrate and boiled for $10 \mathrm{~min}$. Two milliliters of boiling supernatant were reacted with $2 \mathrm{~mL}$ of glacial acetic acid and $2 \mathrm{~mL}$ of ninhydrin and boiled for $30 \mathrm{~min}$. When the temperature of the reaction mixture reached room temperature, it was extracted with $4 \mathrm{~mL}$ of toluene, and the supernatant was measured at $520 \mathrm{~nm}$. At the same time, the same method was used to determine the absorbance values of different standards of proline to construct a standard curve. Finally, the proline content was calculated according to the standard curve. 


\section{Conclusions}

In this study, the P5CS gene cloned from S. purpurea had the same structure as the P5CS genes of other plant species. The expression level of SpP5CS was significantly upregulated under drought stress conditions, and SpP5CS localized to the cytoplasm. The overexpression of SpP5CS in A. thaliana increased the drought resistance and root lengths, and it reduced damage to the cell membrane. We speculate that, under drought stress conditions, the SpP5CS expression level increases, which further increases the proline contents of plant cells, thereby improving the drought resistance of the plants.

Author Contributions: Y.Y. (Yongping Yang) and Y.Y. (Yunqiang Yang) designed the experiments. D.Y., S.Y., M.Q. and Y.P. performed the experiments. D.Y. and R.N. analyzed the data and wrote the paper. Y.Y. (Yongping Yang) revised the manuscript. All authors have read and agreed to the published version of the manuscript.

Funding: This research was funded by the National Natural Science Foundation of China (grant number 41771123 and 32070362); the Yunnan Fundamental Research Projects (grant number 202101AT070190); Digitalization, Development and Application of Biotic Resource (grant number 202002AA100007); poverty alleviation through science and technology projects of the Chinese Academy of Sciences (grant number KFJ-FP-201905) and technology transfer into the Yunnan Project (grant number 202003AD150005).

Institutional Review Board Statement: Not applicable.

Informed Consent Statement: Not applicable.

Data Availability Statement: Not applicable.

Conflicts of Interest: The authors declare no conflict of interest.

\section{References}

1. Geng, G.; Wu, J.; Wang, Q.; Lei, T.; He, B.; Li, X.; Mo, X.; Luo, H.; Zhou, H.; Liu, D. Agricultural drought hazard analysis during 1980-2008: A global perspective. Int. J. Climatol. 2016, 36, 389-399. [CrossRef]

2. Basu, S.; Ramegowda, V.; Kumar, A.; Pereira, A. Plant adaptation to drought stress. F1000Research 2016, 5, 1554. [CrossRef] [PubMed]

3. Hassan, N.; El-bastawisy, Z.; Ebeed, H.; Nemat Alla, M.N. Role of defense enzymes, proteins, solutes and $\Delta 1-$ pyrroline-5carboxylate synthase in wheat tolerance to drought. Rend. Fis. Acc. Lincei 2015, 26, 281-291. [CrossRef]

4. Verbruggen, N.; Hermans, C. Proline accumulation in plants: A review. Amino Acids 2008, 35, 753-759. [CrossRef] [PubMed]

5. Ebeed, H.T.; Hassan, N.M.; Keshta, M.M.; Hassanin, O.S. Comparative analysis of seed yield and biochemical attributes in different sunflower genotypes under different levels of irrigation and salinity. Egypt. J. Bot. 2019, 59, 339-355. [CrossRef]

6. Ebeed, H.T.; Stevenson, S.R.; Cuming, A.C.; Baker, A. Conserved and differential transcriptional responses of peroxisome associated pathways to drought, dehydration and ABA. J. Exp. Bot. 2018, 69, 4971-4985. [CrossRef]

7. Ebeed, H.T.; Hassan, N.M.; Aljarani, A.M. Exogenous applications of Polyamines modulate drought responses in wheat through osmolytes accumulation, increasing free polyamine levels and regulation of polyamine biosynthetic genes. Plant Physiol. Biochem. 2017, 118, 438-448. [CrossRef] [PubMed]

8. Szabados, L.; Savouré, A. Proline: A multifunctional amino acid. Trends Plant Sci. 2010, 15, 89-97. [CrossRef]

9. Rehman, A.U.; Bashir, F.; Ayaydin, F.; Kota, Z.; Pali, T.; Vass, I. Proline is a quencher of singlet oxygen and superoxide both in in vitro systems and isolated thylakoids. Physiol. Plant. 2021, 172, 7-18. [CrossRef]

10. Venekamp, J. Regulation of cytosol acidity under conditions of drought. Physiol. Plant. 2006, 76, 112-117. [CrossRef]

11. Hare, P.D.; Cress, W.A. Metabolic implications of stress-induced proline accumulation in plants. Plant Growth Regul. 1997, 21, 79-102. [CrossRef]

12. $\mathrm{Hu}, \mathrm{C}$. A.; Delauney, A.J.; Verma, D.P. A bifunctional enzyme (delta 1-pyrroline-5-carboxylate synthetase) catalyzes the first two steps in proline biosynthesis in plants. Proc. Natl. Acad. Sci. USA 1992, 89, 9354-9358. [CrossRef] [PubMed]

13. Bandurska, H.; Niedziela, J.; Pietrowska-Borek, M.; Nuc, K.; Chadzinikolau, T.; Radzikowska, D. Regulation of proline biosynthesis and resistance to drought stress in two barley (Hordeum vulgare L.) genotypes of different origin. Plant Physiol. Biochem. 2017, 118, 427-437. [CrossRef] [PubMed]

14. Igarashi, Y.; Yoshiba, Y.; Sanada, Y.; Yamaguchi-Shinozaki, K.; Wada, K.; Shinozaki, K. Characterization of the gene for $\Delta 1$ pyrroline-5-carboxylate synthetase and correlation between the expression of the gene and salt tolerance in Oryza sativa L. Plant Mol. Biol. 1997, 33, 857-865. [CrossRef] 
15. Parida, A.K.; Dagaonkar, V.S.; Phalak, M.S.; Aurangabadkar, L.P. Differential responses of the enzymes involved in proline biosynthesis and degradation in drought tolerant and sensitive cotton genotypes during drought stress and recovery. Acta Physiol. Plant. 2008, 30, 619-627. [CrossRef]

16. Chakraborty, K.; Sairam, R.K.; Bhattacharya, R.C. Salinity-induced expression of pyrrolline-5-carboxylate synthetase determine salinity tolerance in Brassica spp. Acta Physiol. Plant. 2012, 34, 1935-1941. [CrossRef]

17. Zhang, C.-S.; Lu, Q.; Verma, D.P.S. Removal of feedback inhibition of $\Delta 1$-pyrroline-5-carboxylate synthetase, a bifunctional enzyme catalyzing the first two steps of proline biosynthesis in plants. J. Biol. Chem. 1995, 270, 20491-20496. [CrossRef]

18. Xue, X.; Liu, A.; Hua, X. Proline accumulation and transcriptional regulation of proline biosynthesis and degradation in Brassica napus. BMB Rep. 2009, 42, 28-34. [CrossRef]

19. Chen, J.B.; Yang, J.W.; Zhang, Z.Y.; Feng, X.F.; Wang, S.M. Two P5CS genes from common bean exhibiting different tolerance to salt stress in transgenic Arabidopsis. J. Genet. 2013, 92, 461-469. [CrossRef]

20. Hur, J.; Jung, K.-H.; Lee, C.-H.; An, G. Stress-inducible OsP5CS2 gene is essential for salt and cold tolerance in rice. Plant Sci. 2004, 167, 417-426. [CrossRef]

21. Wei, C.; Cui, Q.; Zhang, X.-Q.; Zhao, Y.-Q.; Jia, G.-X. Three P5CS genes including a novel one from Lilium regale play distinct roles in osmotic, drought and salt stress tolerance. J. Plant Biol. 2016, 59, 456-466. [CrossRef]

22. Kim, G.-B.; Nam, Y.-W. A novel $\Delta 1$-pyrroline-5-carboxylate synthetase gene of Medicago truncatula plays a predominant role in stress-induced proline accumulation during symbiotic nitrogen fixation. J. Plant Physiol. 2013, 170, 291-302. [CrossRef]

23. $\mathrm{Su}, \mathrm{J} . ; \mathrm{Wu}, \mathrm{R}$. Stress-inducible synthesis of proline in transgenic rice confers faster growth under stress conditions than that with constitutive synthesis. Plant Sci. 2004, 166, 941-948. [CrossRef]

24. Yamchi, A.; Rastgar Jazii, F.; Mousavi, A.; Karkhane, A.A.; Renu. Proline accumulation in transgenic tobacco as a result of expression of Arabidopsis $\Delta$ 1-pyrroline-5-carboxylate synthetase (P5CS) during osmotic stress. J. Plant Biochem. Biotechnol. 2007, 16, 9-15. [CrossRef]

25. Vendruscolo, E.C.G.; Schuster, I.; Pileggi, M.; Scapim, C.A.; Molinari, H.B.C.; Marur, C.J.; Vieira, L.G.E. Stress-induced synthesis of proline confers tolerance to water deficit in transgenic wheat. J. Plant Physiol. 2007, 164, 1367-1376. [CrossRef] [PubMed]

26. Julia, G.; Nathalia, B.; Rosângela, M.; Andrea, H.; Douglas, D.; Joao, B.; Luiz, V. Stress-induced $\Delta 1$-pyrroline-5-carboxylate synthetase (P5CS) gene confers tolerance to salt stress in transgenic sugarcane. Acta Physiol. Plant. 2014, 36, 2309-2319. [CrossRef]

27. Yang, Y.; Dong, C.; Yang, S.; Li, X.; Sun, X.; Yang, Y. Physiological and proteomic adaptation of the alpine grass Stipa purpurea to a drought gradient. PLoS ONE 2015, 10, e0117475. [CrossRef]

28. Chen, J.; Zhang, X.; Jing, R.; Blair, M.W.; Mao, X.; Wang, S. Cloning and genetic diversity analysis of a new P5CS gene from common bean (Phaseolus vulgaris L.). Theor. Appl. Genet. 2010, 120, 1393-1404. [CrossRef]

29. Su, M.; Li, X.-F.; Ma, X.-Y.; Peng, X.-J.; Zhao, A.-G.; Cheng, L.-Q.; Chen, S.-Y.; Liu, G.-S. Cloning two P5CS genes from bioenergy sorghum and their expression profiles under abiotic stresses and MeJA treatment. Plant Sci. 2011, 181, 652-659. [CrossRef]

30. Szekely, G.; Ábrahám, E.; Cséplö, A.; Rigó, G.; Zsigmond, L.; Csiszár, J.; Ayaydin, F.; Strizhov, N.; Jasik, J.; Schmelzer, E.; et al. Duplicated P5CS genes of Arabidopsis play distinct roles in stress regulation and developmental control of proline biosynthesis. Plant J. 2007, 53, 11-28. [CrossRef]

31. Qin, L.; Chen, E.; Li, F.; Yu, X.; Liu, Z.; Yang, Y.; Wang, R.; Zhang, H.; Wang, H.; Liu, B.; et al. Genome-wide gene expression profiles analysis reveal novel insights into drought stress in foxtail millet (Setaria italica L.). Int. J. Mol. Sci. 2020, 21, 8520. [CrossRef] [PubMed]

32. Silva-Ortega, C.O.; Ochoa-Alfaro, A.E.; Reyes-Agüero, J.A.; Aguado-Santacruz, G.A.; Jiménez-Bremont, J.F. Salt stress increases the expression of $p 5 c s$ gene and induces proline accumulation in cactus pear. Plant Physiol. Biochem. 2008, 46, 82-92. [CrossRef]

33. Rodrigues, T.d.S.; Lins, J.T.; Cattem, M.V.; Jardim, V.C.; Buckeridge, M.S.; Grossi-de-Sá, M.F.; Reinert, F.; Alves-Ferreira, M. Evaluation of Setaria viridis physiological and gene expression responses to distinct water-deficit conditions. Biotech. Res. Innov. 2019, 3, 42-58. [CrossRef]

34. Cao, L.; Han, L.; Zhang, H.-1.; Xin, H.-b.; Imtiaz, M.; Yi, M.-F.; Sun, Z.-Y.; Ju, G.-S.; Qian, Y.-Q.; Liu, J.-X. Isolation and characterization of pyrroline-5-carboxylate synthetase gene from perennial ryegrass (Lolium perenne L.). Acta Physiol. Plant. 2015, 37, 62. [CrossRef]

35. Bajji, M.; Kinet, J.-M.; Lutts, S. The use of the electrolyte leakage method for assessing cell membrane stability as a water stress tolerance test in durum wheat. Plant Growth Regul. 2002, 36, 61-70. [CrossRef]

36. Yang, Y.; Li, X.; Kong, X.; Ma, L.; Hu, X.; Yang, Y. Transcriptome analysis reveals diversified adaptation of Stipa purpurea along a drought gradient on the Tibetan Plateau. Funct. Integr. Genom. 2015, 15, 295-307. [CrossRef]

37. Li, X.; Yang, Y.; Yang, S.; Sun, X.; Yin, X.; Zhao, Y.; Yang, Y. Comparative proteomics analyses of intraspecific differences in the response of Stipa purpurea to drought. Plant Divers. 2016, 38, 101-117. [CrossRef]

38. Zhou, Y.; Sun, X.; Yang, Y.; Li, X.; Cheng, Y.; Yang, Y. Expression of Stipa purpurea SpCIPK26 in Arabidopsis thaliana Enhances Salt and Drought Tolerance and Regulates Abscisic Acid Signaling. Int. J. Mol. Sci. 2016, 17, 966. [CrossRef]

39. Zhao, M.-r.; Li, F.; Fang, Y.; Gao, Q.; Wang, W. Expansin-regulated cell elongation is involved in the drought tolerance in wheat. Protoplasma 2011, 248, 313-323. [CrossRef] [PubMed]

40. Larkin, M.A.; Blackshields, G.; Brown, N.P.; Chenna, R.; McGettigan, P.A.; McWilliam, H.; Valentin, F.; Wallace, I.M.; Wilm, A.; Lopez, R.; et al. Clustal W and Clustal X version 2.0. Bioinformatics 2007, 23, 2947-2948. [CrossRef] 
41. Kumar, S.; Stecher, G.; Li, M.; Knyaz, C.; Tamura, K. MEGA X: Molecular evolutionary genetics analysis across computing platforms. Mol. Biol. Evol. 2018, 35, 1547-1549. [CrossRef] [PubMed]

42. Livak, K.J.; Schmittgen, T.D. Analysis of relative gene expression data using Real-Time quantitative PCR and the $2^{-\Delta \Delta C T}$ method. Methods 2001, 25, 402-408. [CrossRef] [PubMed]

43. Clough, S.J.; Bent, A.F. Floral dip: A simplified method for Agrobacterium-mediated transformation of Arabidopsis thaliana. Plant J. 1998, 16, 735-743. [CrossRef] [PubMed]

44. Yoo, S.D.; Cho, Y.H.; Sheen, J. Arabidopsis mesophyll protoplasts: A versatile cell system for transient gene expression analysis. Nat. Protoc. 2007, 2, 1565-1572. [CrossRef]

45. Kalhor, M.S.; Aliniaeifard, S.; Seif, M.; Asayesh, E.J.; Bernard, F.; Hassani, B.; Li, T. Enhanced salt tolerance and photosynthetic performance: Implication of $\gamma$-amino butyric acid application in salt-exposed lettuce (Lactuca sativa L.) plants. Plant Physiol. Biochem. 2018, 130, 157-172. [CrossRef]

46. Guan, C.; Cui, X.; Liu, H.Y.; Li, X.; Li, M.Q.; Zhang, Y.W. Proline biosynthesis enzyme genes confer salt tolerance to switchgrass (Panicum virgatum L.) in cooperation with polyamines metabolism. Front. Plant Sci. 2020, 11, 46. [CrossRef] 